\title{
Del derecho consuetudinario a la justiciabilidad de los derechos indígenas. El legado de Rodolfo Stavenhagen a la antropología jurídica
}

\author{
MARÍA TERESA SIERRA CAMACHO
}

From Customary Law to the

Justiciability of Indigenous Rights. Rodolfo Stavenhagen's Legacy to Legal Anthropology

María Teresa Sierra Camacho Centro de Investigaciones y Estudios Superiores en Antropología SocialCiudad de México, México mtsierrac@hotmail.com

Desacatos 57, mayo-agosto 2018, pp. 156-165 odolfo Stavenhagen fue un defensor incansable de los derechos de los pueblos indígenas y un académico de primer nivel, comprometido con desvelar las estructuras de la desigualdad social y el racismo estructural y colonial de nuestras sociedades. Estas preocupaciones se hicieron visibles en particular en uno de los campos de investigación que tomó gran parte de sus energías durante más de 30 años y hasta el final de sus días: el campo del derecho indígena y los derechos humanos.

Como lo hizo en otras áreas, Rodolfo Stavenhagen fue un innovador y sentó las bases de lo que hoy es la antropología jurídica latinoamericana: una antropología crítica y comprometida, de corte interdisciplinario, volcada al estudio de las configuraciones socioculturales del derecho y en especial del pluralismo jurídico como una realidad constitutiva de la relación entre el Estado y los pueblos indígenas. Con base en la perspectiva analítica de sus trabajos seminales sobre el colonialismo interno y la teoría de la dependencia, incorporó una mirada relacional y de poder al estudio del derecho consuetudinario indígena con el fin de analizar las formas de la regulación social que han construido los pueblos indígenas en el contexto de una historia de dominación y exclusión ante los Estados nacionales (Stavenhagen, 1981). Esta perspectiva se distingue de las visiones funcionalistas que concibieron el derecho consuetudinario fuera de la historia y del poder, y de los estudios de corte evolutivo que lo ubicaron como expresión atávica de costumbres premodernas (Sierra y Chenaut, 2002).

En este texto me interesa destacar las contribuciones de Rodolfo Stavenhagen al desarrollo de la antropología jurídica latinoamericana y mostrar los debates fundantes de este campo y sus desarrollos posteriores a partir de la mirada crítica y comprometida que impulsó. Sus conceptualizaciones son referentes clave para el estudio contemporáneo del derecho indígena y del sentido que asumen las luchas indígenas frente 
al Estado en los actuales contextos de globalización y violencia neoliberal. Para ello me valgo de mi propia experiencia de investigación como integrante de un grupo de trabajo sobre derecho consuetudinario indígena creado por Stavenhagen y hago referencia a problemas que han nutrido a la Red Latinoamericana de Antropología Jurídica (Relaju), ${ }^{1}$ que reconoce en él a uno de sus precursores.

\section{El derecho consuetudinario indígena en Latinoamérica. Una agenda de investigación}

En julio de 1987, hace ya 30 años, Rodolfo Stavenhagen convocó a un grupo de académicos de varias instituciones y disciplinas con el fin de impulsar investigaciones comparativas sobre el derecho consuetudinario indígena en México y Latinoamérica. ${ }^{2}$ En un estudio previo sobre legislaciones en la región y el tratamiento de la cuestión indígena, documentó el desconocimiento del derecho consuetudinario en las leyes nacionales, lo que estaba detrás de una violación sistemática a los derechos humanos de los indígenas, en especial cuando se enfrentaban con la justicia estatal (Stavenhagen, 1987). Esto coincidía con los resultados de un estudio pionero mundial, el llamado Informe Martínez Cobos (Martínez, 1983), elaborado por el guatemalteco Augusto Willemsen Díaz, en un momento en que se buscaba abrir el foro mundial de la Organización de las Naciones Unidas (ONU) al reconocimiento de la discriminación y la violación sistemática a los derechos de las poblaciones originarias. Este informe hacía referencia a la vigencia de órdenes jurídicos en sociedades indígenas y nativas colonizadas con los cuales regulaban su vida. Los órdenes, sin embargo, eran negados de manera sistemática en las leyes nacionales. Si bien los estudios clásicos de la antropología anglosajona, incluso en México, habían dado testimonio de la vigencia del derecho consuetudinario en sociedades indígenas (Sierra y Chenaut, 2002), ninguno de estos estudios — si bien pioneros - priorizaba la conexión entre los ordenamientos indígenas y la conformación de los Estados nacionales. No consideraban el sentido en que la situación colonial y étnica de un gran número de poblaciones reproducía la condición de desigualdad y las exclusiones, lo que se traducía en una violación sistemática a sus derechos humanos, en particular en el ámbito laboral. Por esta razón, resultaba de gran interés documentar el derecho consuetudinario indígena y su relación con el derecho estatal, y alimentar con ello el debate internacional sobre los derechos colectivos de los pueblos indígenas, en un momento en el que el movimiento indígena se organizaba en el continente para cuestionar el colonialismo y defender una agenda propia, a finales de la década de 1980. Estas constataciones fueron la base para impulsar un proyecto de investigación latinoamericano en el que por primera vez se comparaban los ordenamientos jurídicos que organizaban la vida de comunidades originarias en la mayor parte de los países del subcontinente americano, cuyo resultado fue el libro coordinado por Rodolfo Stavenhagen y Diego Iturralde (1990). Además de esto, Stavenhagen impulsaba otro gran proyecto de investigación sobre conflictos étnicos en el mundo (Stavenhagen, 1996) y participaba en el Grupo de Trabajo sobre Poblaciones Indígenas y Tribales de la ONU. Los debates de este grupo fueron esenciales para avanzar en el reconocimiento de los derechos colectivos de los pueblos indígenas, que llevaron a modificar el

1 La Relaju se creó en 1997, en la ciudad de Quito, Ecuador, como un espacio de investigación y promoción de los derechos indígenas y el pluralismo jurídico en Latinoamérica. El X Encuentro Internacional tendrá lugar en octubre de 2018, en Temuco, Chile, en homenaje a Rodolfo Stavenhagen.

2 El grupo comenzó con la participación de Stavenhagen, Magdalena Gómez, Diego Iturralde, Victoria Chenaut, Enrique Hamel, François Lartigue, Deborah Dorotinsky y quien suscribe. 
Convenio 169 de la Organización Internacional del Trabajo (OIT, 1989) y después impulsarían una ola de reformas en el constitucionalismo latinoamericano en un horizonte de pluralidad (Yrigoyen, 2011).

La capacidad de Rodolfo Stavenhagen de moverse en distintos planos nacionales e internacionales y de articular redes académicas, funcionarios públicos, defensores de derechos humanos y legisladores, además de generar diálogos y una fuerte empatía con las organizaciones indígenas, fue central para avanzar hacia una agenda por el reconocimiento de los derechos indígenas. Esta agenda se benefició del desarrollo de trabajos de investigación en torno al derecho propio y el pluralismo jurídico como base de las demandas autonómicas (Iturralde, 1990). De esta manera, se sentaron las bases de la antropología jurídica latinoamericana.

\section{Las apuestas por los derechos humanos de los pueblos indígenas. El derecho propio y el pluralismo jurídico}

La agenda de investigación de este grupo de trabajo buscaba documentar los órdenes jurídicos indígenas y sus formas de relación y conflicto con el derecho estatal, lo cual implicaba destacar un hecho invisibilizado: que las comunidades indígenas contaban con sistemas jurídicos propios y no sólo "costumbres" atrasadas proclives a violar derechos. Quienes participamos en este grupo retomamos la propuesta de Stavenhagen de hacer un análisis relacional y de poder, al estudiar los órdenes jurídicos indígenas y considerar las dinámicas de cambio legal y el sentido en que el acceso a la justicia estatal beneficiaba o perjudicaba a los indígenas (Stavehagen e Iturralde, 1990; Chenaut

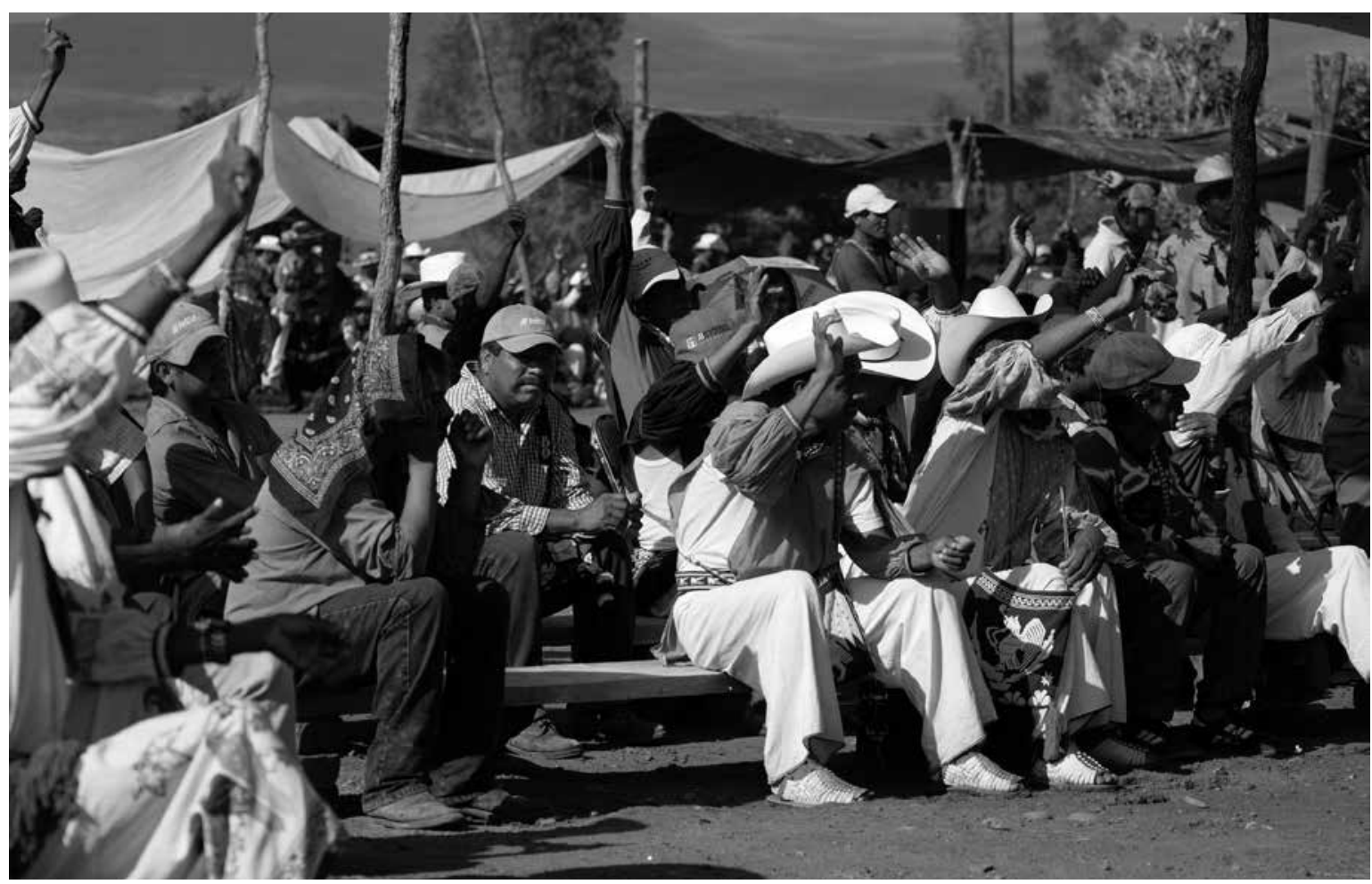

PROMETEO LUCERO La asamblea general de la comunidad wixárika de San Sebastián Teponahuaxtlán reitera su rechazo a la minería en su territorio. Mesa del Tirador, Jalisco, 2012. 
y Sierra, 1995). El objetivo último era documentar, a partir de las realidades etnográficas, la particularidad del derecho consuetudinario indígena y las afectaciones a los derechos humanos que se generaba cuando los indígenas accedían a la justicia estatal.

\section{Entre la ley y la costumbre}

Las investigaciones del grupo de trabajo tuvieron dos direcciones principales: por un lado, estudiar las formas propias del derecho consuetudinario para dar testimonio de su vitalidad sin dejar de mostrar su relación con el Estado, y por el otro, los escenarios y prácticas del enfrentamiento del indígena con la justicia estatal en los que se hacían evidentes los mecanismos de la discriminación social y el racismo judicial.

\section{DERECHO CONSUETUDINARIO INDÍGENA}

En su contribución al libro colectivo, Stavenhagen avanzó en la definición del derecho consuetudinario indígena y planteó cuatro aspectos centrales: 1) concebir el derecho como un elemento fundamental de la estructura social de un pueblo y un elemento básico de su identidad; 2) considerar la dimensión integral del derecho propio que se opone a plantear lo jurídico como un campo separado de lo social; 3) tratar los derechos colonizados en continua relación con el derecho estatal, lo que cuestiona las visiones aislacionistas, autocontenidas del derecho propio, y 4) el reto metodológico de estudiar un derecho oral, no escrito, difícil de asir por no encontrarse definido de antemano (Stavenhagen, 1990). Dado que se trata de un derecho no escrito, la forma privilegiada para documentar el derecho consuetudinario ha sido el estudio de caso, por lo general a partir del seguimiento de disputas. El caso constituye una unidad de análisis accesible en la medida en que involucra no sólo mecanismos de resolución de controversias sino también el recurso a principios y valores que regulan los comportamientos de una colectividad en contextos específicos. Ésa ha sido la tradición de la antropología jurídica anglosajona, que tuvo una importante influencia en nuestros trabajos (Sierra y Chenaut, 2002). No es mi propósito referirme a la variedad de corrientes de la antropología jurídica, ${ }^{3}$ sino destacar los desafíos que enfrentamos como etnógrafas al querer reconstruir los sistemas jurídicos indígenas en la perspectiva relacional y de poder planteada por Stavenhagen.

Un gran reto metodológico en el estudio de la justicia indígena ha sido subrayar la tensión entre normas y prácticas como un referente productivo para analizar el deber ser, propio de normas y valores, y la distancia que suele haber con las acciones que producen los conflictos en situaciones concretas. Esta tensión era aprovechada por las autoridades comunitarias al momento de dirimir las controversias. Por ejemplo, en mi experiencia de investigación, al dar seguimiento a juicios en comunidades nahuas de Huachinango, en la Sierra Norte de Puebla (Sierra, 1995a; 1995b), salía a relucir cómo las autoridades — jueces o principales - apelaban a principios y valores para juzgar los comportamientos y evidenciar los agravios cometidos por las partes involucradas en una disputa, fueran vecinos o familiares. Para ello, recurrían a estrategias diversas que llamaban a buscar el entendimiento y algún tipo de acuerdo. Pude observar largas sesiones de conciliación en lengua nahua, por lo regular públicas, con presencia de familiares, que podían durar uno o varios días, sobre asuntos que no involucraban lesiones graves. Resultaba evidente la capacidad retórica de la autoridad para llegar a arreglos no necesariamente equitativos, que solían acompañarse de una disculpa o petición de perdón del inculpado hacia la víctima, hecho que 
resultaba de gran relevancia. Así descubrí cómo las normas, en tanto principios del deber ser, se hacían evidentes en el momento mismo de dirimir las disputas, no como códigos abstractos que norman el comportamiento, sino como lenguajes que permiten valorar acciones concretas que provocaban agravios y se consideraban ofensas o delitos (Collier, 1995). De esta manera, más que evaluar si se cumplía o no una determinada norma, lo que llamaba la atención eran los procesos para llegar a acuerdos y lo que las personas identificaban como agravios. En estas dinámicas emergían principios y valores en juego. Las comunidades se constituían así como campos sociales semiautónomos (Moore, 1973), que mostraban una dinámica normativa y de regulación propia, la cual, sin embargo, tenía una importante interacción con la justicia estatal, pues los límites de la autoridad comunitaria quedaban claros. Esta justicia sólo atendía asuntos considerados no graves, sin involucrar hechos llamados de sangre. Es decir, se trataba de una justicia marginal, permitida, en la medida en que se adecuaba a lo establecido en un marco legal estatal que no necesitaba reconocer la justicia comunitaria para dejar espacios para su vigencia en asuntos considerados menores.

Éstos fueron algunos de los procedimientos por medio de los cuales buscamos reconstruir los sistemas normativos indígenas, lo que revelaba entramados normativos involucrados en lógicas culturales y de poder que resultaban eficaces para dirimir cierto tipo de asuntos, en el marco de su subordinación al derecho estatal.

\section{INDÍGENAS Y ACCESO A LA JUSTICIA}

El trabajo de Magdalena Gómez al frente del programa de defensoría jurídica del Instituto Nacional Indigenista (INI), a finales de la década de 1980, contribuyó de manera relevante a construir la otra cara de la moneda del derecho consuetudinario, cuando los indígenas terminaban presos, sujetos a la justicia estatal. El programa de defensoría se dio a la tarea de apoyar legalmente a los indígenas en prisión, en las regiones en las que la institución tenía presencia. A partir del seguimiento de algunos casos, Gómez ilustró las violaciones a los derechos humanos más elementales que vivían los indígenas sujetos a proceso: se les juzgaba en una lengua que no dominaban, sobre delitos establecidos en una ley que no contemplaban sus propias normas y solían ser sentenciados a largas penas de encierro, que muchas veces no correspondían con el delito cometido (Gómez, 1990). Ya fueran indígenas rarámuris, zapotecos, nahuas o tsotsiles, se repetía una historia de agravios y obstáculos que los abogados del INI buscaban sortear con estrategias jurídicas para bajar la pena o liberar al inculpado, pero no para ejecutar una defensa jurídica justa. De esta manera, la defensa de un rarámuri acusado de homicidio llevó a los abogados a utilizar el argumento sobre la incapacidad penal del detenido y no a exponer el contexto que condujo a la comisión del delito y los motivos culturales involucrados, es decir, el recurso legal implicaba usar argumentos que inferiorizaban al indígena y lo situaban como incapaz para apelar la decisión del juez. Por ello, según Gómez (1990), de manera sistemática se imponía la verdad jurídica sobre la verdad real o histórica, cada vez más distanciadas en el proceso judicial. Con esto se refería al contexto estructural de exclusión que enfrentan los indígenas en su paso por la justicia, es decir, un racismo judicial (Escalante, 2015).

\section{Del derecho consuetudinario al derecho indígena. El debate contemporáneo}

El concepto de derecho consuetudinario y el de costumbre jurídica — que utilizamos para poner énfasis en el dinamismo de las normas comunitarias- pronto fueron desechados para dar lugar a 


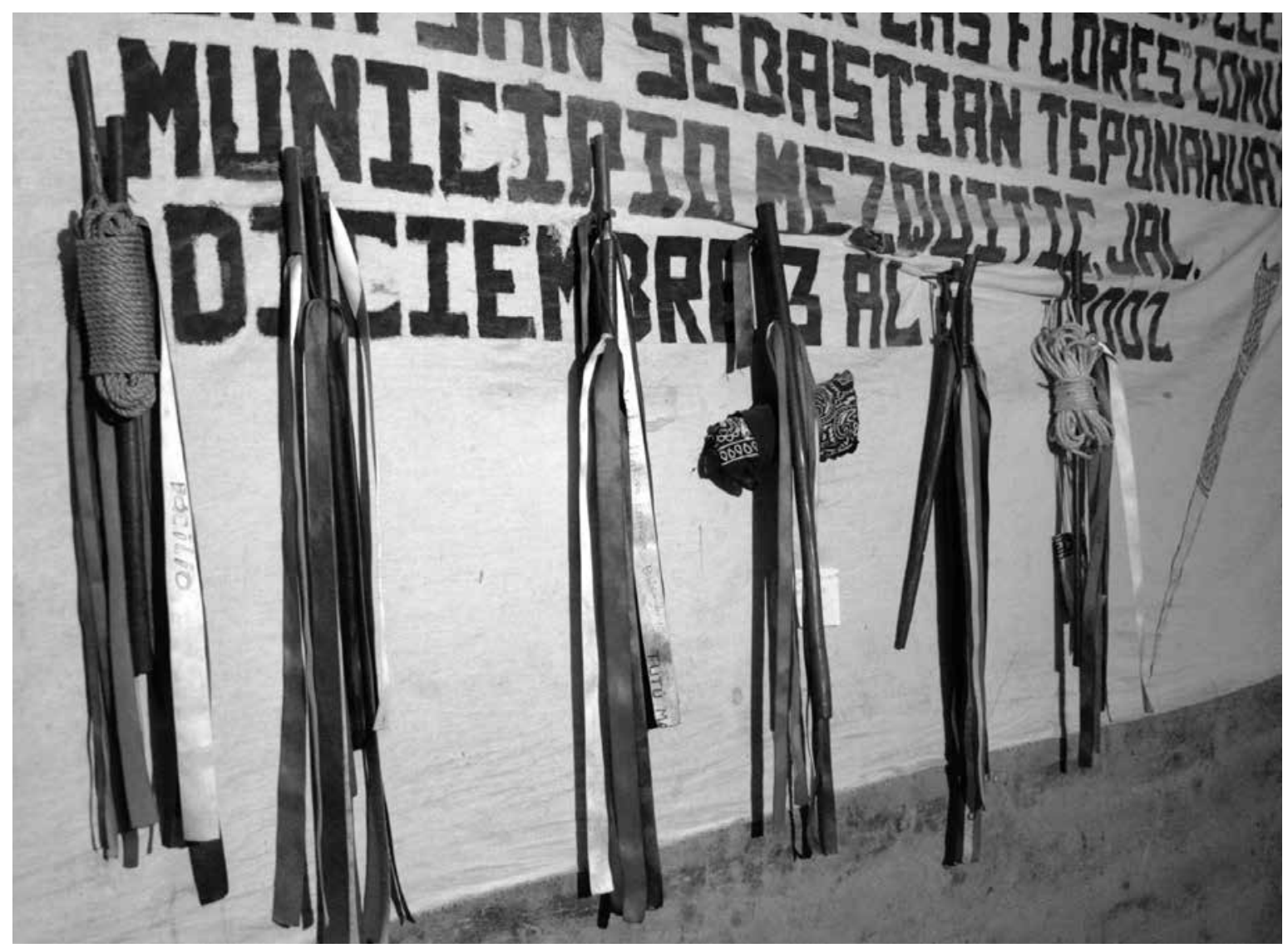

YURI ESCALANTE V Varas de mando huichol, Mezquitic, Jalisco, 2012.

importantes debates que recogimos en varias publicaciones (Chenaut y Sierra, 1992; Valdivia, 1992, Sierra, 1993; Gómez, 1997). El cuestionamiento al concepto tenía que ver con que involucraba una acepción inacabada del derecho indígena al nombrarlo como consuetudinario, lo que implicaba reproducir su condición de "no derecho" ante el derecho estatal. Sobre todo, era producto de la posición de los propios indígenas, que no vacilaron en referirse a sus sistemas jurídicos en toda la extensión de la palabra, es decir, con autoridades, procedimientos y normas de regulación, lo que legitimó el uso del término derecho indígena. No obstante, en una perspectiva antropológica, emergían otros problemas al documentar no tanto la sistematicidad del sistema jurídico indígena sino los usos del derecho en sus prácticas. Veíamos con reserva las propuestas que terminaban construyendo el derecho indígena con el modelo positivo del derecho estatal para sistematizar sus normas y jerarquías. Se hacía evidente una tensión entre, por un lado, un acercamiento procesual del derecho indígena que daba cuenta de su expresión material en prácticas y representaciones, y por el otro, visiones normativas que en ocasiones terminaban proponiendo su codificación. Tales planteamientos revelaban también el dilema académico y político de abstraer los sistemas normativos indígenas de su contexto y los riesgos de su esencialización, al mismo tiempo que se hacía evidente la necesidad de legitimar el derecho 
indígena frente a su descalificación en contextos de movilización política (Sierra, 1997). Debates similares sucedieron en otros espacios al discutir los efectos del colonialismo y la globalización sobre los sistemas jurídicos indígenas y los derechos humanos (Starr y Collier, 1989; Sieder, 1996).

Los dilemas que enfrentamos en esas épocas en torno a la conceptualización del derecho indígena siguen presentes en la actualidad y asumen nuevos significados, sobre todo cuando intervenimos en peritajes antropológicos como antropólogos jurídicos para hacer relevante la diferencia cultural en procesos judiciales. Entre otros puntos, nos preguntamos: ¿es posible avanzar en conceptualizaciones dinámicas del derecho propio que destaquen el particularismo cultural sin negar su historicidad y capacidad de adecuación a nuevas realidades? ¿En qué sentido las cosmovisiones indígenas pueden ser traducidas al lenguaje jurídico que tiende a fragmentar y simplificar sus estructuras para hacerlas legibles al juzgador? Esas conceptualizaciones pueden interpretarse hoy en el marco crítico de la colonialidad del poder (Quijano, 2000), es decir, desde una visión que deconstruye la producción del conocimiento hegemónico, que en este caso ha implicado la imposición de ideologías racializadas que inferiorizan los sistemas normativos indígenas como un subderecho. Esto sucede con el concepto de "usos y costumbres", incorporado en la judicialización de los derechos indígenas y utilizado también por los actores indígenas. El concepto revela las condiciones estructurales de subordinación a partir de las cuales los indígenas construyen sus reclamos.

Los desarrollos actuales de la antropología jurídica, al documentar las formas contemporáneas del derecho indígena y su materialidad en el campo de la justicia, contribuyen a desestabilizar las nociones hegemónicas del derecho y a disputar su definición del derecho en los espacios mismos de la producción jurídica, en especial en lo que concierne a la diferencia cultural y los derechos humanos, como puede verse en el acompañamiento de casos que involucran la defensa de jurisdicciones indígenas.

\section{Garantías de acceso a la justicia y la justiciabilidad de los derechos indígenas}

En este apartado me refiero a un campo de gran vitalidad, en el que la antropología jurídica ha tenido un papel activo en relación con la judicialización de casos que han llegado a las altas Cortes de Justicia nacionales e internacionales, que involucran demandas de gran calado para los pueblos indígenas, en defensa de sus derechos colectivos, en particular territoriales y de sus recursos naturales. Rodolfo Stavenhagen fue un defensor activo de estos procesos, siempre del lado de los pueblos, ya sea ante las instancias internacionales, o bien, ante los gobiernos de los países involucrados en violaciones graves a los derechos de los pueblos indígenas. Sobre todo en su papel de primer relator por los Derechos Humanos de los Pueblos Indígenas de la ONU (2001-2008), Stavenhagen desarrolló un activismo político que le permitió hacer diagnósticos y entrevistarse con funcionarios, gobernantes, organizaciones indígenas y de la sociedad civil para impulsar la agenda indígena en diversas partes del mundo, donde fue convocado de visita oficial. Por ejemplo, cuando visitó Chile como relator, en 2003, abogó ante el gobierno del país contra la criminalización de indígenas mapuches presos — considerados terroristas - y en defensa de la propiedad comunal de la tierra y la territorialidad indígena. Su recomendación contribuyó a la ratificación del Convenio 169 (OIT, 1989), que ocurrió en 2008. En su primera visita oficial como relator en Guatemala, en 2002, retrató en su informe la violencia política y la represión de Estado que provocó el genocidio del pueblo maya. Gracias a uno de estos informes como relator, la Corte Interamericana de Derechos 
Humanos adoptó el estándar del "consentimiento" como requisito previo para que el Estado otorgue concesiones en territorios de pueblos indígenas o tribales, como en el caso de la sentencia del pueblo saramaka vs. Surinam (CIDH, 2007). Según lo narra Stavenhagen en un texto autoetnográfico, la experiencia como relator de los Derechos Humanos de los Pueblos Indígenas de la ONU si bien le ofreció una oportunidad única para hacer evidentes las violaciones graves a los derechos humanos sobre los indígenas y exigir compromisos a los Estados, también le hizo ver los límites de estas intervenciones en el más alto nivel. Recojo sus palabras por su elocuencia para dar testimonio de lo que significó para él asumir el papel de relator:

Me han acompañado dos frustraciones en esta experiencia. La primera, que los informes preparados por el relator no se transforman ipso facto en mejora visible de la situación de los derechos humanos de los pueblos indígenas y que sus recomendaciones no sean implementadas de manera puntual por los gobiernos y el sistema internacional [...]. Con todo, para numerosos pueblos indígenas los informes del relator constituyen un aliciente en la continua lucha por sus derechos humanos, y para los gobiernos un referente contra el cual pueden ser evaluadas las acciones emprendidas (2015: 24).

Esta tarea le dio a Rodolfo grandes enseñanzas y satisfacciones, y le permitió también mostrar los claroscuros del activismo judicial y destacar lo que identificó como los grandes escollos a la justiciabilidad de los derechos: la brecha de la implementación, con la cual se refirió a la distancia que revela la matriz del poder colonial y neoliberal que impide la puesta en práctica de los derechos indígenas.

Dejaba claro que no bastan los cambios legislativos, por más avanzados que sean, si no se consigue la fuerza para implementarlos. Stavenhagen apuntaba a una de las grandes quimeras que viven los movimientos indígenas que discuten los límites de la acción jurídica. No por ello dejó de apelar a soluciones políticas y negociadas con los poderes establecidos, sin dejar de señalar los grandes obstáculos para garantizar a los indígenas el acceso a la justicia del Estado.

\section{Conclusiones}

El campo de la antropología jurídica latinoamericana ha evolucionado de manera importante a lo largo de estos 30 años, desde que Rodolfo Stavenhagen impulsó las investigaciones sobre derecho consuetudinario indígena hacia áreas más allá del problema étnico, para comprender las configuraciones socioculturales del derecho en contextos de violencia neoliberal y de violación sistemática a los derechos humanos de poblaciones diversas, excluidas y subordinadas. No obstante, los debates fundantes relativos al estudio del pluralismo jurídico y el derecho indígena siguen marcando buena parte de las discusiones contemporáneas, dado que apuntan a problemas que siguen vigentes e inspiran nuevas líneas de investigación. Stavenhagen contribuyó a plantear de manera crítica rutas que abonan al reconocimiento y la defensa de los derechos humanos de los pueblos indígenas y otros colectivos subordinados.

Stavenhagen fue pionero y visionario en el campo de las ciencias humanas. Mantuvo una ética y un compromiso constante con los pueblos indígenas y la defensa de sus derechos humanos contra toda forma de exclusión, subordinación y racismo, fue, en efecto, un académico y activista de amplio espectro. La antropología jurídica latinoamericana se ha beneficiado ampliamente de sus contribuciones. Una manera de honrar su memoria es seguir avanzando en las investigaciones que aporten al campo de la justiciabilidad de los derechos indígenas y a la lucha contra la exclusión y el racismo. D 


\section{Bibliografía}

Chenaut, Victoria y María Teresa Sierra, 1992, "El campo de investigación de la antropología jurídica”, en Nueva Antropología, vol. 13, núm. 43, pp. 101-109.

_ (coords.), 1995, Pueblos indigenas ante e/ derecho, Centro de Investigaciones y Estudios Superiores en Antropología Social/Centro de Estudios Mexicanos y Centroamericanos, México.

Collier, Jane, 1995 [1973], El derecho zinacanteco, Centro de Investigaciones y Estudios Superiores en Antropología Social/Universidad de Ciencias y Artes de Chiapas, México.

Corte Interamericana de Derechos Humanos (CIDH), 2007, "Caso del pueblo saramaka vs. Surinam. Sentencia del 28 de noviembre de 2007 (excepciones preliminares, fondo, reparaciones y costas)". Disponible en línea: <http://www.corteidh.or.cr/docs/casos/articulos/seriec_172_esp.pdf>. Consultado el 29 de marzo de 2018.

Escalante Betancourt, Yuri, 2015, El racismo judicial en México, Juan Pablos, México.

Gómez, Magdalena, 1990, “La defensoría jurídica de presos indígenas”, en Rodolfo Stavenhagen y Diego Iturralde (comps.), Entre la ley y la costumbre, Instituto Indigenista Interamericano, México, pp. 371-388.

__ (coord.), 1997, Derecho indígena. Instituto Nacional Indigenista/Asociación Mexicana para las Naciones Unidas, México.

__ 2013, "Los pueblos indígenas y la razón de Estado en México: elementos para un balance”, en Nueva Antropología, vol. 26, núm. 78, pp. 43-62.

Iturralde, Diego, 1990, "Movimiento indio, costumbre jurídica y usos de la ley", en Rodolfo Stavenhagen y Diego Iturralde (comps.), Entre la ley y la costumbre, Instituto Indigenista Interamericano, México, pp. 47-63.

Krotz, Esteban (ed.), 2002, Antropología jurídica. Perspectivas socioculturales en el estudio del derecho, Anthropos/Universidad Autónoma Metropolitana-Iztapalapa, Barcelona y México.

Martínez Cobos, José, 1983, "Estudio del problema de la discriminación contra las poblaciones indígenas. Informe final (última parte)", Naciones Unidas-Consejo Económico y Social, 30 de septiembre. Disponible en línea: <http://indigenas.bioetica.org/not/MCS_xxi_ xxii_s.pdf>. Consultado el 24 de agosto de 2017.

Moore, Sally Falk, 1973, Law as Process, Routledge \& Kegan Paul, Londres.

Organización Internacional del Trabajo (OIT), 1989, “Convenio sobre Pueblos Indígenas y Tribales, 1989 (núm. 169)”. Disponible en línea: <http:// www.ilo.org/dyn/normlex/es/f?p=NORMLEXPUB:12100:0::NO::P12100_INSTRUMENT_ID:312314>. Consultado el 29 de marzo de 2018.

Quijano, Aníbal, 2000, “Colonialidad del poder, eurocentrismo y América Latina”, en Edgardo Lander (comp.), La colonialidad del saber: eurocentrismo y ciencias sociales. Perspectivas latinoamericanas, Consejo Latinoamericano de Ciencias Sociales, Buenos Aires.

Sieder, Rachel, 1996, Derecho consuetudinario y transición democrática en Guatemala, Facultad Latinoamericana de Ciencias Sociales, Guatemala.

Sierra, María Teresa, 1993, "Usos y desusos del derecho consuetudinario indígena”, en Nueva Antropología, núm. 44, pp. 17-26.

, 1995a, "Indian Rights and Customary Law in Mexico: A Study of the Nahuas in the Sierra de Puebla", en Law \& Society Review, vol. 29 núm. 2, pp. 227-254.

, 1995b, "Articulaciones entre ley y costumbre: estrategias jurídicas de los nahuas", en Victoria Chenaut y María Teresa Sierra (coords.), Pueblos indígenas ante el derecho, Centro de Investigaciones y Estudios Superiores en Antropología Social/Centro de Estudios Mexicanos y Centroamericanos, México, pp. 101-123.

, 1997, “Esencialismo y autonomía. Paradojas de las reivindicaciones indígenas”, en Alteridades, vol. 7, núm. 14, pp. 131-143.

Sierra, María Teresa y Victoria Chenaut, 2002, "Debates recientes y actuales de la antropología jurídica. Las corrientes anglosajonas”, en Esteban Krotz (ed.), Antropología jurídica. Perspectivas socioculturales en el estudio del derecho, Anthropos/Universidad Autónoma Metropolitana-Iztapalapa, Barcelona y México, pp. 113-170

Starr, June y Jane Collier (eds.), 1989, History and Power in the Study of Law. New Directions in Legal Anthropology, Cornell University Press, Ithaca.

Stavenhagen, Rodolfo, 1981 [1970], "Siete tesis equivocadas sobre América Latina”, en Rodolfo Stavenhagen, Sociología y subdesarrol/o, Nuestro Tiempo, México.

, 1987, Derecho indígena y derechos humanos en América Latina, El Colegio de México/Instituto Indigenista Interamericano de Derechos Humanos, México. 
_ 1990, "Derecho consuetudinario indígena en América Latina”, en Rodolfo Stavenhagen y Diego Iturralde (coords.), Entre la ley y la costumbre, Instituto Indigenista Interamericano, México, pp. 27-46.

_ 1996, Ethnic Conflicts and The Nation-State, MacMillan, Londres.

2007, Los pueblos indigenas y sus derechos. Informes temáticos del relator especial sobre la situación de los Derechos Humanos y las Libertades Fundamentales de los Pueblos Indigenas del Consejo de Derechos Humanos de la Organización de las Naciones Unidas, Organización de las Naciones Unidas para la Educación, la Ciencia y la Cultura, México.

2015, "Etnografía activista. Mi experiencia en la onu”, en Nueva Antropología, vol. 28, núm. 83, pp. 13-24.

Stavenhagen, Rodolfo y Diego Iturralde (coords.), 1990, Entre la ley y la costumbre, Instituto Indigenista Interamericano, México.

Valdivia, Teresa, 1992, “¿Por qué hoy una antropología jurídica en México?”, en Nueva Antropología, vol. 13, núm. 43, pp. 111-122.

Yrigoyen Fajardo, Raquel, 2011, “El horizonte del constitucionalismo pluralista: del multiculturalismo a la descolonización”, en César RodríguezGaravito (coord.), El derecho en América Latina: un mapa para el pensamiento jurídico del siglo XXI, Siglo XXI Editores, Buenos Aires. 